\title{
Investigation of the Psycho-Social Factors Affecting the Adoption of a Healthy Nutrition Style in Women with a History of Gestational Diabetes Using Structural Equation Modeling
}

\author{
Banafsheh Mohammadi Zeidi1 ${ }^{1}$, Nourossadat Kariman ${ }^{2,}$, Zahra Kashi ${ }^{3}$, Isa Mohammadi zeidi ${ }^{4}$ and Hamid Alavi \\ Majd $^{5}$ \\ ${ }^{1}$ Student Research Committee, Department of Midwifery and Reproductive Health, Faculty of Nursing and Midwifery, Shahid Beheshti University of Medical \\ Sciences, Tehran, Iran \\ 2 Midwifery and Reproductive Health Research Center, Midwifery and Reproductive Health Department, Faculty of Nursing and Midwifery, Shahid Beheshti \\ University of Medical Sciences, Tehran, Iran \\ 3 Diabetes Research Center, Mazandaran University of Medical Sciences, Sari, Iran \\ ${ }^{4}$ Department of Public Health, Faculty of Health, Qazvin University of Medical Sciences, Qazvin, Iran \\ ${ }^{5}$ Department of Biostatistics, Paramedical School, Shahid Beheshti University of Medical Sciences, Tehran, Iran
}

* Corresponding author: Nourossadat Kariman, Midwifery and Reproductive Health Research Center, Midwifery and Reproductive Health Department, Faculty of Nursing and Midwifery, Shahid Beheshti University of Medical Sciences, Tehran, Iran. Tel: +982188202512; Email: n_kariman@sbmu.ac.ir

Received 2020 August 14; Revised 2020 September 11; Accepted 2020 0ctober 18.

\begin{abstract}
Background: Women with a history of gestational diabetes mellitus are the main high-risk group for type 2 diabetes; however, a healthy nutrition style can reduce the risk of developing diabetes in this group.

Objectives: The present study aimed to determine the psycho-social factors that affect the adoption of a healthy nutrition style in women with a history of gestational diabetes.

Methods: This cross-sectional study was conducted based on the Health Action Process Approach (HAPA) in the west of Mazandaran province, Iran in 2019. A total of 165 women with a history of gestational diabetes in a recent pregnancy were selected using the convenience sampling method. The required data were collected using a demographic characteristic form, a healthy nutrition style questionnaire, and a researcher-made questionnaire based on HAPA model constructs. Moreover, structural equation modeling was used for data analysis.

Results: Based on the results, the data were fit to the model (Tucker-Lewis index $=0.924$, comparative fit index $=0.928$, root mean square error of approximation $=0.045, \chi^{2} /$ degrees of freedom $=1.332$ ). The model constructs predicted $23 \%$ and $51 \%$ of intention variance and nutrition style variance, respectively. Action self-efficacy and risk perception were the most important predictors of intention. In addition, planning and recovery self-efficacy significantly predicted a healthy nutrition style.

Conclusion: As the first step, using the HAPA for the prediction of the nutrition style of women with a history of gestational diabetes was confirmed. Therefore, this model can be used to design educational interventions to prevent diabetes.

Keywords: Gestational diabetes, Healthy diet, Postpartum, Psychosocial factors
\end{abstract}

\section{Background}

Gestational Diabetes Mellitus (GDM) is one of the most common endocrine disorders during pregnancy (1). The GDM is diagnosed in almost one in five pregnancies according to the International Association of the Diabetes and Pregnancy Study Groups (2). Mostly, the hyperglycemia caused by this disorder vanishes immediately after the delivery (3); however, women with a history of GDM are exposed to a high risk of developing type 2 diabetes. Approximately, 50\% of them will develop type 2 diabetes during the first five years after delivery (4).

A healthy diet is the key to the prevention of chronic diseases, such as diabetes. All women with a history of GDM should have a balanced diet, eat fruits or vegetables at least five times a day, and reduce their salt, sugar, and fat intake based on the recommendation of the American College of
Obstetricians and Gynecologists and the American Diabetes Association (5). However, the results of the studies performed in Australia (6), the Netherlands (7), the United States (8-10), and Sweden (11) have indicated that the diet quality of most women with a history of gestational diabetes was lower than the optimal level (10), suggesting the need to develop health promotion interventions (12).

Development of such interventions requires identification of the behavioral determinants that are changeable and can be included in the intervention. In order to identify these determinants, researchers use theories of behavioral science and psychology (13). In many theories, the intention for change is the best predictor of real change; however, people often do not act on their intentions since sometimes unpredicted obstacles arise, and sometimes, people succumb to their temptations. Therefore, to facilitate the conversion of intention into behavior, the presence of other factors, such as self-efficacy and 
strategic plans, is essential. These factors help bridge the gap between intention and behavior (14).

This gap is also taken into account in the theory of the Health Action Process Approach (HAPA). In this theory, it is assumed the formation of the intention for doing a behavior is affected by the action self-efficacy, the outcome expectancies, and the risk perception. After the formation of intention, factors, such as action and coping planning as well as maintenance self-efficacy turn the intention into behavior. Recovery self-efficacy will also help maintain behavior $(14,15)$.

Few studies have been published on the psychosocial factors that affect the nutritional behavior of women with a history of gestational diabetes. The results obtained by Zehle et al. in 2008 (16) revealed that self-efficacy is a predictor of fruit and vegetable consumption. Ferranti et al. (9) also found that there is a significant relationship between self-efficacy and diet quality in 2014. In a previous study by Kaiser et al. carried out in 2016 (5), social support was the strongest predictor of a healthy diet.

In Iran, no study has yet been conducted to investigate the factors affecting the diet of women with a history of GDM. Furthermore, there is no standard HAPA-based instrument that can measure these factors in women with a history of gestational diabetes in Iran. Number of studies performed on the nutrition of women with a history of GDM is limited and there is a need to test theories of behavior change before using them in educational interventions to identify their most important constructs. Therefore, it is important to conduct research on this issue.

\section{Objectives}

The present study aimed to determine the psychosocial factors that affect healthy nutrition in women with a history of gestational diabetes.

\section{Methods}

The present descriptive cross-sectional study was conducted during 2019 in Mazandaran province in Iran. In this study, the sample size was estimated using the software introduced by Westland $(17,18)$. This software calculates the minimum sample size for structural equation modeling (SEM) based on the number of observable and latent variables in the model, the anticipated effect size, the probability, and the desired statistical power level. Therefore, with 49 observable and 9 latent variables and considering the effect size 0.5 and the statistical power 0.8 , the minimum sample size required for this study at the confidence level of 0.95 was calculated at 133 subjects. This effect size was based on the medium effect size reported by Gollwitzer and Sheeran in
2006 (19).

For the purposes of the study, four cities were randomly selected for sampling. Afterward, several urban and rural health centers were randomly selected from each city. Subsequently, 192 women with a history of gestational diabetes were identified in the selected centers. However, five of them were excluded due to developed type 2 diabetes and the other eligible women were invited to participate in the study. Participants were selected from these women using the convenience sampling method and 22 eligible women were reluctant to participate in the study.

The inclusion criteria included 1) Iranian nationality, 2) history of GDM in a recent pregnancy, 3 ) passage of at least 6 and at most 24 months from childbirth, 4) lack of pregnancy or intention of it in the next 6 months, and 5) willingness to participate in the study. The exclusion criteria included chronic diseases (e.g., type 1 or type 2 diabetes, high blood pressure, depression, and cancer), smoking habits, and substance abuse.

\subsection{Data collection}

The required data were collected using a demographic and clinical profile questionnaire, a selfreport researcher-made questionnaire to evaluate HAPA model constructs, and a nutrition style questionnaire.

\subsection{Self-report researcher-made questionnaire encompassing Health Action Process Approach constructs}

This questionnaire included 31 items and was designed based on Schwarzer's recommendation, as the designer of this theory (20), and a literature review. It should be mentioned that two items of this questionnaire were taken from a study conducted by PinidiaPatridge (2018) (21) which were scored based on a five-point Likert scale ranging from one (very low) to five (very high). These two items measured the risk perception construct.

Moreover, five, three, two, and four items of this questionnaire measured the outcome expectancies construct, intention, and action and coping planning, respectively. These questions were obtained from the reviews of Ferranti (2014) (14), Ajzan (2006) (22), and Renner (2008) (23), respectively, and scored according to the five-point Likert scale ranging from 1 (strongly disagree) to five (strongly agree).

Furthermore, six, three, and six items measured action self-efficacy constructs according to Ochsner's study (2013) (24), recovery self-efficacy according to Rouhani's study (2016)(25), and the maintenance self-efficacy construct based on the review of studies that addressed barriers of healthy nutrition in women with a history of gestational diabetes $(12,26,27)$, respectively. The items of these three constructs scored according to the five-point Likert 
scale ranging from one (not sure at all) to five (completely sure).

The content validity ratio and content validity index of the final questionnaire were estimated at 0.94 and 0.98, respectively. In this study, Cronbach's alpha for the whole questionnaire was calculated at 0.855 (0.823-0.866) and the total intraclass correlation was 0.824 (0.712-0.940) (Table 2).

\subsection{Nutrition Style questionnaire}

In the present study, nutritional behavior was evaluated using a questionnaire with 18 items on nutrition style. This questionnaire was designed in 2006 by Lippke and Ziegelmann and scored according to the four-point Likert scale. This questionnaire was localized in Iran by Rouhani and his colleagues (28).

When the participants were completing the questionnaires, a member of the research team was present at the site to emphasize the confidentiality of their information, answer their possible questions, and make sure the questions were answered accurately and completely.

\subsection{Ethical considerations}

The study was approved by the Research Ethics Committee of Shahid Beheshti University of Medical Sciences, Tehran, Iran (code of ethics: IR.SBMU. RETECH.REC.1397.831).

\subsection{Data analysis}

The collected data were analyzed in IBM SPSS software (version 23) using descriptive analysis (i.e., frequency, percentages, and means). Afterward, the Pearson correlation test was used to determine the association between HAPA constructs and the nutrition style. The SEM with latent variable was used to test the hypothesized models. In the present study, the covariance matrix was considered as the maximum likelihood estimation. The univariate normality was evaluated using skewness and kurtosis. Moreover, it should be noted that the distribution of all variables was normal. In addition, Mardia's test was performed to evaluate the distribution of multivariate normality. The results revealed non-normality in a few variables; however, since the maximum likelihood estimation procedure was robust to modest, the violations of multivariate normality were considered appropriate and the analysis was continued (29).

The most important indices of model adequacy assessment were chi-squared to the degree of freedom $\left(\chi^{2} / \mathrm{df}\right)$, the root mean square error of approximation (RMSEA), the comparative fit index (CFI), the Tucker-Lewis index (TLI), and the incremental fit index (IFI). In the present study, $\chi^{2} /$ df less than 3, RMSEA less than 0.8, and CFI, TLI, and IFI greater than 0.9 indicated the model fit to the data (30).

\section{Results}

Out of all the eligible women in the statistical population, 165 women were included in this study who were with the age range of 19-48 years. Table 1 tabulates the other demographic and clinical characteristics of the participants. The nutrition style score of only $25.5 \%$ of them was at the desired level. The mean value of the nutrition style and other HAPA model constructs are summarized in Table 2.

According to table 3 , the Pearson correlation test results of HAPA model constructs indicated that the intention construct has a positive and significant correlation with the three constructs of risk perception, outcome expectancies, and action selfefficacy. Among these three constructs, the strongest significant correlation coefficient was observed between action self-efficacy and intention $(r=0.427$, $\mathrm{P}<0.001$ ). The nutrition style also had a positive and significant correlation with all HAPA model constructs except the risk perception construct $(\mathrm{r}=0.107, \mathrm{P}=0.173)$. Among the HAPA model constructs, the coping planning construct had the strongest significant correlation coefficient with the nutrition style $(\mathrm{r}=0.508, \mathrm{P}<0.001)$.

Fit of the primary model was unsatisfactory $(\chi 2 / \mathrm{df}=1.83, \mathrm{RMSEA}=0.071, \mathrm{CFI}=0.802, \mathrm{IFI}=0.808$, TLI=0.792). Given the strong correlation between action planning and coping planning $(r=0.582)$, these two constructs were considered as a single planning construct to obtain a better model. The obtained model is shown in Figure 1using this new construct. Moreover, it is noteworthy that the fit indices of the final model were satisfactory $(\chi 2 / \mathrm{df}=1.332$, RMSEA=0.045, CFI=0.928, $\mathrm{IFI}=0.928, \mathrm{TLI}=0.924$ ).

Table 1. Demographic and clinical characteristics of the participants

\begin{tabular}{lc}
\hline Variable & Mean (SD) \\
\hline Age & $33.35 \pm 5.22$ \\
\hline Time since the last GDM pregnancy (months) & $13.19 \pm 6.39$ \\
Variable & $\begin{array}{c}\text { Frequency } \\
\text { (percent) }\end{array}$ \\
Educational level & $121(73.3 \%)$ \\
$\quad$ Diploma or below diploma degree & $44(26.7 \%)$ \\
Academic degree & \\
Place of residence & $75(45.5 \%)$ \\
City & $90(54.5 \%)$ \\
Village & $137(83 \%)$ \\
Employment status & $28(17 \%)$ \\
Housewife & \\
Employed & $47(28.5 \%)$ \\
Parity & $118(71.5 \%)$ \\
$\quad$ Primipara & \\
Multipara & $107(64.8 \%)$ \\
Family history of diabetes & $58(35.2 \%)$ \\
yes & \\
$\quad$ No & $30(18.2 \%)$ \\
Body mass index & $135(81.8 \%)$ \\
$\quad$ Normal-weight & \\
Overweight or obese & \\
\hline GDM: gestational diabetes mellitus &
\end{tabular}


Table 2. Mean, Standard Deviation, Cronbach's alpha, and intraclass correlation of the health action process approach constructs

\begin{tabular}{|c|c|c|c|c|}
\hline Construct & No of items & Means (SD) & Cronbach's alpha & Intraclass correlation \\
\hline Risk perception & 2 & $3.18 \pm 1.19$ & 0.866 & 0.844 \\
\hline Outcome expectancies & 5 & $3.97 \pm 077$ & 0.854 & 0.866 \\
\hline Action S.E. & 6 & $3.24 \pm 1.10$ & 0.838 & 0.759 \\
\hline Intention & 3 & $288 \pm 1.26$ & 0.844 & 0.826 \\
\hline Action planning & 2 & $2.88 \pm 1.26$ & 0.845 & 0.940 \\
\hline Coping planning & 4 & $3.25 \pm 1.08$ & 0.823 & 0.868 \\
\hline Maintenance S.E. & 6 & $2.82 \pm 1.15$ & 0.831 & 0.751 \\
\hline Recovery S.E. & 3 & $3.36 \pm 1.30$ & 0.844 & 0.927 \\
\hline Nutrition style & 18 & $47.74 \pm 9.28$ & 0.849 & 0.712 \\
\hline
\end{tabular}

S.E.: Self-efficacy

Table 3. Correlation of variable used in the model

\begin{tabular}{|c|c|c|c|c|c|c|c|c|c|c|}
\hline & HAPA construct & $\mathbf{1}$ & 2 & 3 & 4 & 5 & 6 & 7 & 8 & 9 \\
\hline 1 & Risk perception & 1 & & & & & & & & \\
\hline 2 & Outcome Expectations & 0.136 & 1 & & & & & & & \\
\hline 3 & Action S.E. & 0.110 & $0.259 * *$ & 1 & & & & & & \\
\hline 4 & Intention & $0.209^{* *}$ & $0.292^{* *}$ & $0.427 * *$ & 1 & & & & & \\
\hline 5 & Action planning & 0.021 & $0.219^{* *}$ & $0.456^{* *}$ & $0.339 * *$ & 1 & & & & \\
\hline 6 & Coping planning & 0.104 & $0.302^{* *}$ & $0.459^{* *}$ & $0.415^{* *}$ & $0.582^{* *}$ & 1 & & & \\
\hline 7 & Maintenance S.E. & 0.065 & $0.297 * *$ & $0.501^{* *}$ & $0.372^{* *}$ & $0.808^{* *}$ & $0.725^{* *}$ & 1 & & \\
\hline 8 & Recovery S.E. & 0.074 & $0.282^{* *}$ & $0.395^{* *}$ & $0.381^{* *}$ & $0.400^{* *}$ & $0.873^{* *}$ & $0.504^{* *}$ & 1 & \\
\hline 9 & Nutrition style & 0.107 & $0.293^{* *}$ & $0.402^{* *}$ & $0.407^{* *}$ & $0.370^{* *}$ & $0.508^{* *}$ & $0.465^{* *}$ & $0.455^{* *}$ & 1 \\
\hline
\end{tabular}

HAPA: health action process approach, S.E.: self-efficacy, $* * \mathrm{P}=0.01$

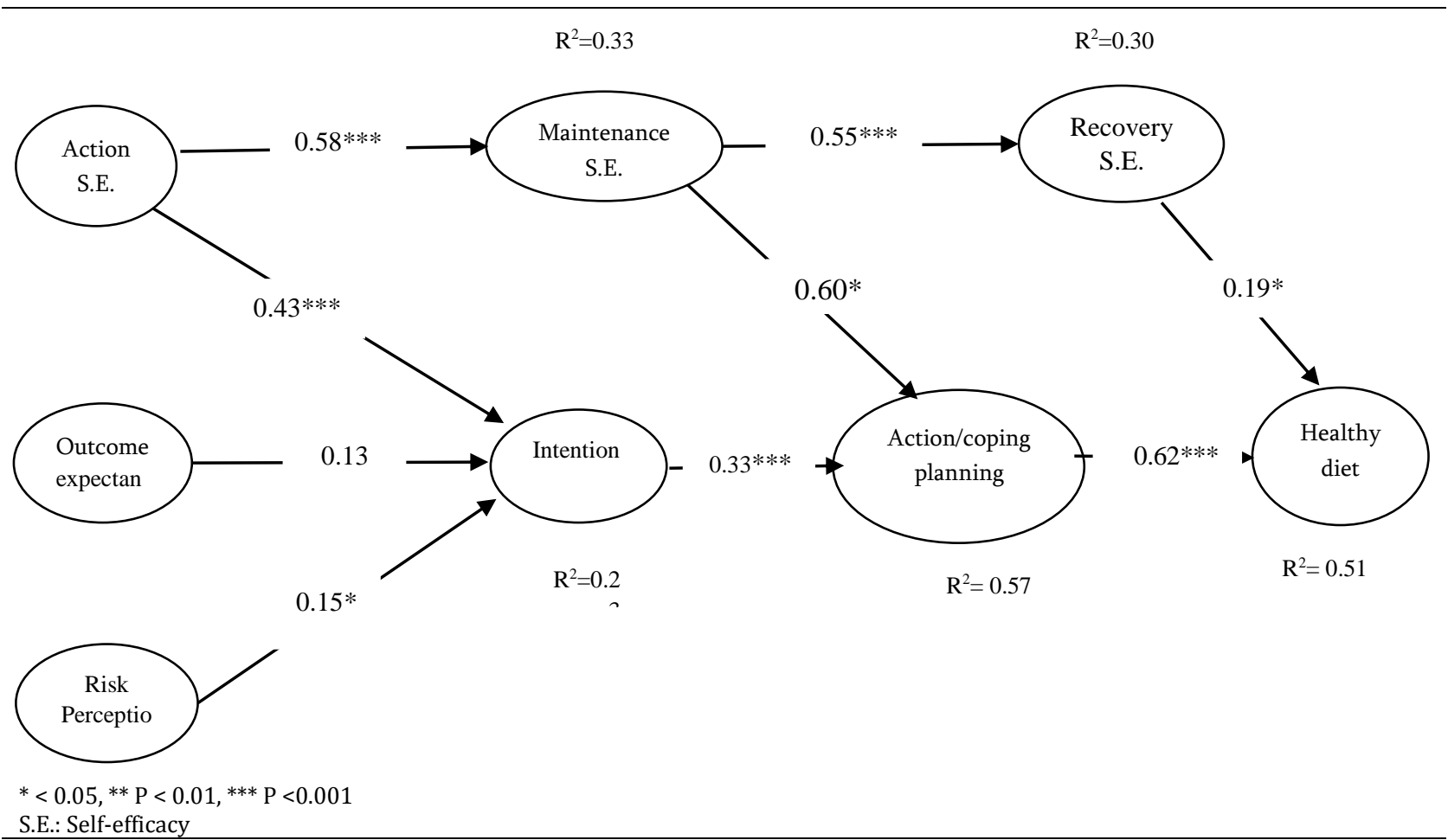

Figure 1. Structural model for the prediction of physical activity behavior using the health action process approach model

Based on the results of SEM (Figure 1), in the motivational phase, action self-efficacy $(\beta=0.43$, $\mathrm{P}<0.001)$ and the risk perception $(\beta=0.15, \mathrm{P}=0.026)$, except for the outcome expectancies $(\beta=0.13$, $P=0.097$ ), had a significant relationship with the intention to adopt a healthy nutrition style. Accordingly, these three constructs explained 23\% of the intention variance. In the voluntary phase, maintenance self-efficacy $(\beta=0.60, \quad P<0.001)$ and intention $(\beta=0.33, \quad \mathrm{P}<0.001)$ had a significant association with the planning (a combination of the two constructs of action and coping planning); accordingly, $57 \%$ of the variance of the planning construct was explained by these two constructs. Furthermore, the planning $(\beta=0.62, P<0.001)$ and the recovery self-efficacy $(\beta=0.19, P=0.018)$ were significantly related to the healthy nutrition style. Constructs of this model explained $51 \%$ of the 
variance of a healthy nutrition style.

\section{Discussion}

The present study aimed to find the psycho-social factors that affect the nutritional behavior of women with a history of GDM. Based on the results, the nutrition style score of the majority of participants was undesirable. This finding was consistent with those of the studies conducted by Morrison et al. in 2012 (6), Ferranti et al. in 2014 (9), Xiao et al. in 2015 (10), and Kaiser et al. in 2016 (5). Since women with a history of GDM are the main risk groups for type 2 diabetes (31), such results should be considered a serious warning and the necessary interventions should be designed to improve their nutritional behavior.

In the present study, action self-efficacy was the strongest predictor variable of intent. This finding was consistent with those of the research performed by Schwarzer in 2007 (32), Chiu in 2010 (33), Zhang in 2018 (15), Rouhani in 2018 (25), and Teleki in 2018 (34). This finding indicated that one's belief in her abilities is the most important factor in shaping intention. it also implied that in health-promoting interventions, to increase the score of intention and the possibility of adoption of a healthy nutrition style, it is necessary to improve action self-efficacy through various means, such as education, establishment of achievable goals, role-play, and positive reinforcement (14).

In many studies, the risk perception construct did not have a significant effect on the prediction and modification of behavior $(13,33-35)$; however, in the present study, the predictive power of the risk perception construct was significant. A similar finding was observed in the studies carried out by Renner in 2008 (23) and Chiu in 2012 (33). This result can be justified by the fact that the history of gestational diabetes in participants increased the perceived sensitivity to type 2 diabetes, as a result, they are more inclined to adopt a healthy nutrition style.

Unlike previous studies, in this study, the willingness of the subjects to adopt a healthy diet was not greatly affected by the outcome expectancies. However, using only three questions may not fully reflect the outcome expectancies of the adoption of a healthy diet and may lead to a poor relationship between the outcome expectancies and intentions (36). The findings also indicated that mere awareness of the benefits of a healthy diet cannot lead to the willingness for the adoption of a healthy diet in women with a history of gestational diabetes. Moreover, it was found that the existence of two other constructs, namely, action self-efficacy and risk perception are also essential for the formation of such an intention (19).

Consistent with other studies $(23,33,37)$, in the present study, the action self-efficacy predicted maintenance self-efficacy which was, in turn, a predictor of recovery self-efficacy and planning. This finding points out that the maintenance self-efficacy is a mediator factor between action self-efficacy and recovery self-efficacy and between action self-efficacy and planning. These relationships are also logical in theory since people who want to do something will face a lot of unpredictable obstacles. Self-confidence to deal with these obstacles creates the necessary motivation to try harder and continue the desired behavior (23). These optimistic beliefs lead to planning in people with higher self-efficacy (38). Development of a plan to deal with potential problems, in addition to initiating a behavior, will also prevent that behavior from being released when faced with obstacles (36).

A review of the previous studies on the use of the HAPA model in the field of nutritional behavior provided contradictory results regarding the relationship between planning and nutritional behavior $(23,33,35,37)$. A possible explanation for this might be that planning is a cognitively demanding self-regulation strategy. The direct relationship observed in the present study may be due to the higher self-regulatory skills of the participants.

In the present study, the planning construct was the strongest predictor of a healthy nutrition style. This finding pointed out that planning was a mediator factor between intention and behavior and suggest that in women with a history of gestational diabetes, planning is essential for turning intention into behavior.

It was the first time that psycho-social factors that affected healthy eating in women at risk of diabetes were evaluated in Iran and can be a starting point for other studies in this field. Usage of a strong statistical method to examine the correlates of healthy eating was another strength of the present study. However, this study also faced several limitations. First, this research was conducted based on a cross-sectional design, in which causal relationships cannot be examined and only a simple relationship can be evaluated. The second limitation was that the nutrition style was assessed through self-reporting tools and the participants might have reported their nutrition style according to the preferences of the community, not as it actually is. To control this limitation, food reminders can be used that must be recorded when every time the person eats.

\section{Conclusion}

As the first step, the present study confirmed that HAPA can be used for the prediction of the nutrition style of women with a history of GDM. Therefore, this model can be used to design educational interventions for the prevention of diabetes in 
women with a history of GDM.

\section{Acknowledgements}

This article was derived from a thesis submitted in partial fulfillment of the requirement for the degree of Doctor of Philosophy in Reproductive Health to Shahid Beheshti University of Medical Sciences. Hereby, the authors express their gratitude to the Research Deputy of the university, all the esteemed personnel of the health centers in the west of Mazandaran province, and all the participating mothers.

\section{Footnotes}

Conflict of Interests: The authors declare that there were no conflicts of interest in this study.

Funding/Support: This study received no specific grant from any funding agency.

\section{References}

1. Behboudi-Gandevani S, Amiri M, Yarandi RB, Tehrani FR. The impact of diagnostic criteria for gestational diabetes on its prevalence: a systematic review and meta-analysis. Diabetol Metab Syndr. 2019;11(1):1-18. doi: 10.1186/s13098-0190406-1. [PubMed: 30733833].

2. O'Reilly S, Versace V, Mohebbi M, Lim S, Janus E, Dunbar J. The effect of a diabetes prevention program on dietary quality in women with previous gestational diabetes. BMC Women's Health. 2019;19(1):1-12.doi:10.1186/s12905-019-0788-0. [PubMed: 31269928].

3. Alfadhli EM. Gestational diabetes mellitus. Saudi Med J. 2015;36(4):399-406. doi:10.15537/smj.2015.4.10307. [PubMed: 25828275].

4. Rayanagoudar G, Hashi AA, Zamora J, Khan KS, Hitman GA, Thangaratinam S. Quantification of the type 2 diabetes risk in women with gestational diabetes: a systematic review and meta-analysis of 95,750 women. Diabetologia. 2016; 59(7):1403-11.doi:10.1007/s00125-016-3927-2. [PubMed: 27073002].

5. Kaiser B, Jeannot E, Razurel C. Determinants of health behaviors after gestational diabetes mellitus: A prospective cohort study in geneva. J Midwifery \& women's health. 2016; 61(5):571-7. doi:10.1111/jmwh.12486. [PubMed: 27434581].

6. Morrison MK, Koh D, Lowe JM, Miller YD, Marshall AL, Colyvas $\mathrm{K}$, et al. Postpartum diet quality in Australian women following a gestational diabetes pregnancy. Eur J Clin Nutr. 2012; 66(10):1160-5. doi:10.1038/ejcn.2012.84. [PubMed: 22781022].

7. Koning SH, Lutgers HL, Hoogenberg K, Trompert CA, van den Berg PP, Wolffenbuttel BH. Postpartum glucose follow-up and lifestyle management after gestational diabetes mellitus: general practitioner and patient perspectives. $J$ Diabetes Metab Disord. 2016;15:1-9. doi: 10.1186/s40200-016-0282-2. [PubMed: 27981042].

8. Bennett WL, Liu S-H, Yeh H-C, Nicholson WK, Gunderson EP, Lewis CE, et al. Changes in weight and health behaviors after pregnancies complicated by gestational diabetes mellitus: the CARDIA Study. Obesity (Silver Spring). 2013;21(6):1269-75. doi: 10.1002/oby.20133. [PubMed: 23666593].

9. Ferranti EP, Narayan KV, Reilly CM, Foster J, McCullough M, Ziegler TR, et al. Dietary self-efficacy predicts AHEI diet quality in women with previous gestational diabetes. Diabetes Educ. 2014;40(5):688-99. doi: 10.1177/0145721714539735. [PubMed: 24942530].

10. Xiao RS, Simas TAM, Person SD, Goldberg RJ, Waring ME. Diet quality and history of gestational diabetes mellitus among childbearing women, united states, 2007-2010. Prev Chronic Dis. 2015;12:1-9. doi: 10.5888/pcd12.140360.[PubMed: 25719215]

11. Persson M, Winkvist A, Mogren I. Lifestyle and health status in a sample of Swedish women four years after pregnancy: a comparison of women with a history of normal pregnancy and women with a history of gestational diabetes mellitus. $B M C$ pregnancy and childbirth. 2015;15(1):1-16. doi: 10.1186/s12884-015-0487-2. [PubMed: 25884665].

12. Razee H, Van Der Ploeg HP, Blignault I, Smith BJ, Bauman AE, McLean $M$, et al. Beliefs, barriers, social support, and environmental influences related to diabetes risk behaviours among women with a history of gestational diabetes. Health Promotion J Austr. 2010;21(2):130-7. doi: 10.1071/he10130. [PubMed: 20701563].

13. Zhang C-Q, Zhang R, Schwarzer R, Hagger MS. A meta-analysis of the health action process approach. Health Psycho. 2019;38(7):623-37. doi: 10.1037/hea0000728. [PubMed: 30973747].

14. Schwarzer R, Luszczynska A. How to overcome healthcompromising behaviors: The health action process approach. Eur Psychol. 2008;13(2):141-51. doi: 10.1027/1016-9040. 13.2.141

15. Zhang C, Zheng X, Huang H, Su C, Zhao H, Yang H, et al. A Study on the Applicability of the Health Action Process Approach to the Dietary Behavior of University Students in Shanxi, China. Nutr Educ Behav. 2018;50(4):388-95. doi: 10.1016/j.jneb. 2017.09.024. [PubMed: 29276018].

16. Zehle K, Smith BJ, Chey T, McLean M, Bauman AE, Wah Cheung N. Psychosocial factors related to diet among women with recent gestational diabetes: opportunities for intervention. Diabetes Educ. 2008;34(5):807-14. doi: 10.1177/0145721708323641. [PubMed: 18832285].

17. A-priori sample size calculator for structural equation models .Software. Available at: URL: http://www. danielsoper. com/statcalc]. 2013;10:2018.

18. Westland JC. Lower bounds on sample size in structural equation modeling. Electron Commer Res Appl. 2010;9(6):47687. doi: 10.1016/j.elerap.2010.07.003.

19. Gollwitzer PM, Sheeran P. Implementation intentions and goal achievement: A meta-analysis of effects and processes. Adv Exp Soc Psychol. 2006;38:69-119. doi: 10.1016/S00652601(06)38002-1.

20. Schwarzer R, Sniehotta F, Lippke S, Luszczynska A, Scholz U, Schüz B, et al. On the assessment and analysis of variables in the health action process approach: Conducting an investigation. Berlin, Germany: Freie Universeitat Berlin. 2003.

21. Pinidiyapathirage J, Jayasuriya R, Cheung NW, Schwarzer R. Self-efficacy and planning strategies can improve physical activity levels in women with a recent history of gestational diabetes mellitus. Psychol health. 2018;33(8):1062-77. doi: 10.1080/08870446.2018.1458983. [PubMed: 29629841].

22. Ajzen I. Perceived behavioral control, self-efficacy, locus of control, and the theory of planned behavior 1. J Appl Soc Psychol. 2002;32(4):665-83. doi: 10.1111/j.1559-1816.2002. tb00236.x.

23. Renner B, Kwon S, Yang BH, Paik KC, Kim SH, Roh S, et al. Social-cognitive predictors of dietary behaviors in South Korean men and women. Int J Behav Med. 2008;15(1):4-13. doi: 10.1007/BF03003068. [PubMed: 18444015].

24. Ochsner S, Scholz U, Hornung R. Testing phase-specific self-efficacy beliefs in the context of dietary behaviour change. Appl Psychol Health Well Being. 2013;5(1):99-117. doi: 10.1111/j.1758-0854.2012.01079.x. [PubMed: 23457086].

25. Rohani H, Bidkhori M, Eslami AA, Sadeghi E, Sadeghi A. Psychological factors of healthful diet promotion among diabetics: an application of health action process approach. Electron Physician. 2018;10(4):6647-54. doi: 10.19082/6647. [PubMed: 29881527].

26. Tierney M, O'Dea A, Danyliv A, Noctor E, McGuire B, Glynn L, et al. Factors influencing lifestyle behaviours during and after a gestational diabetes mellitus pregnancy. Health Psychol Behav Med. 2015;3(1):204-16. doi: 10.1080/21642850.2015.1073111.

27. Nicklas JM, Zera CA, Seely EW, Abdul-Rahim ZS, Rudloff ND, 
Levkoff SE. Identifying postpartum intervention approaches to prevent type 2 diabetes in women with a history of gestational diabetes. BMC pregnancy childbirth. 2011;11(1):1-8. doi: 10.1186/1471-2393-11-23. [PubMed: 21435246].

28. Rohani H, Malekahmadi M, Eslami AA, Ghaderi A, Bidkhori M, Raei $\mathrm{M}$, et al. Predicting dietary behavior of type 2 diabetics: Application of the theory of planned behavior and perceived risk of diabetes complications construct. Chron Dis J. 2016;3(2):71-8. doi: 10.22122/cdj.v3i2.153.

29. Gao S, Mokhtarian PL, Johnston RA. Nonnormality of data in structural equation models. Transp Res Rec. 2008; 2082(1):116-24.

30. Munro BH. Statistical methods for health care research. lippincott williams \& wilkins; 2005.

31. Bellamy L, Casas JP, Hingorani AD, Williams D. Type 2 diabetes mellitus after gestational diabetes: a systematic review and meta-analysis. Lancet. 2009;373(9677):1773-9. doi: 10.1016/S0140-6736(09)60731-5. [PubMed: 19465232].

32. Schwarzer R, Schüz B, Ziegelmann JP, Lippke S, Luszczynska A, Scholz U. Adoption and maintenance of four health behaviors: Theory-guided longitudinal studies on dental flossing, seat belt use, dietary behavior, and physical activity. Ann behav med. 2007;33(2):156-66. doi: 10.1007/BF02879897. [PubMed: 17447868].

33. Chiu CY, Lynch RT, Chan F, Rose L. The health action process approach as a motivational model of dietary self-management for people with multiple sclerosis: A path analysis. Rehabil Counsel Bulletin. 2012;56(1):48-61. doi: 10.1177/003435521 2440888.

34. Teleki S, Zsidó AN, Komócsi A, Lénárd L, Kiss EC, Tiringer I. The role of social support in the dietary behavior of coronary heart patients: an application of the health action process approach. Psychol Health Med. 2019;24(6):714-24. doi: 10.1080/13548506.2018.1550259. [PubMed: 30486665].

35. Rohani H, Eslami AA, Raei M, Jafari Koshki T, Ghaderi A. Evaluation of health action process approach in explaining healthy diet among the patients with Type 2 diabetes. Qom Univ Med Sci J. 2015;9(7):55-64.

36. Zhou G, Gan Y, Ke Q, Knoll N, Lonsdale C, Schwarzer R. Avoiding exposure to air pollution by using filtering facemask respirators: An application of the health action process approach. Health Psychol. 2016;35(2):141-7. doi: 10.1037/ hea0000264. [PubMed: 26371720].

37. ALINAGHIZADEH E, Javadi M, Lesani A. Predicting factors associated with healthy eating nutritional behavior at primary school students in Tehran: an application of the Health Action Process Approach (HAPA). Iranian J Endocrinol Metabol. 2017;19(4):252-60.

38. Richert J, Reuter T, Wiedemann AU, Lippke S, Ziegelmann J, Schwarzer R. Differential effects of planning and self-efficacy on fruit and vegetable consumption. Appetite. 2010;54(3):6114. doi: 10.1016/j.appet.2010.03.006. [PubMed: 20227450]. 